\section{A general analysis of variance program for microcomputers}

\author{
DAVID M. LANE \\ Rice University, Houston, Texas 77001
}

Although other analysis of variance programs have been written for microcomputers, none is capable of analyzing (1) large designs (e.g., four or five factors), (2) designs with unequal cell sizes, and (3) designs with both between- and within-subjects variables. The program described here, in addition to providing these capabilities, computes $\hat{\epsilon}$ for each of the within-subjects effects. As described by Winer (1971), the analysis of within-subjects designs requires assumptions about the covariance matrix $\Sigma$. Although these assumptions are unlikely to hold in practice, failure to meet them has the serious consequence of inflating the Type I error rate. The statistic ê provides a correction for the failure of $\Sigma$ to be of the assumed form.

Limitations. The program can analyze a design with any combination of within- and between-subjects variables as long as the number of independent groups (cells) is not greater than 32, the number of scores per subject is not greater than 32 , and the total number of factors is not greater than nine. Although there is no limit to the number of subjects, there is a combined limit on the number of scores per subject and the number of between-subjects variables. Letting NV be the number of between-subjects variables and $p$ be the number of scores per subject, then $K=\left(2^{N V}+1\right)(p)$ $(p+1) / 2$ has an upper limit. As the program stands, $\mathrm{K}$ must be less than 2,900. For each 4 bytes of additional memory, the limit can be increased by one; this is accomplished by increasing the dimensions of the array A9.

Algorithm. The program computes and stores the hypothesis $(\mathrm{H})$ matrix for each between-subjects effect and the pooled error (E) matrix (see Bock, 1975). Between-subjects effects are calculated directly by summing the elements of the relevant $\mathrm{H}$ matrix. Withinsubjects effects are computed by generating a matrix of contrast coefficients, $W$, for each within-subjects effect. The sum of squares for an effect equals $\left.\mathrm{N}^{\left(\mathrm{W}^{\prime} \mathrm{x}\right.}\right)^{\prime}\left(\mathrm{W}^{\prime} \mathrm{x}\right)$, where $\bar{x}$ is the vector of dependent variable (trial) means and $\mathrm{N}$ is the total number of subjects. The sumof-squares error is simply the trace of $W^{\prime} E W$. The $\hat{\epsilon}$ statistic is also computed from W'EW (see Huynh, 1978). The sum of squares for the interactions of between- and within-subjects variables is equal to the trace of $W^{\prime} \mathrm{HW}$, where $\mathrm{W}$ is the matrix of contrasts representing the relevant within-subjects effect and $\mathrm{H}$ is the hypothesis matrix associated with the relevant betweensubjects effect. Unweighted-means analysis is used in the case of unequal cell sizes.

Computer and Language. The program is written in BASIC and runs on a TRS-80/Model I microcomputer with one disk drive and 32K RAM. A slightly limited version runs in TRS-80 Level II BASIC with 16K RAM. The program should run with most BASICs with only minor modification.

Core and Time Requirements. The program takes up 5,675 bytes in core. An additional 15,304 bytes are used for data storage. The program executes reasonably quickly: It takes $3.25 \mathrm{~min}$ on a TRS- 80 to analyze the data for a 2 by 2 by 3 design with repeated measurements on the last factor and 10 subjects per cell.

Input. The number of subjects per cell (or cell sizes in the case of unequal $\mathrm{n}$ ), the number of betweensubjects variables, the number of within-subjects variables, and the number of levels of each variable are entered on the keyboard. The data can be read in either from the keyboard or from a sequential disk file. The data must be arranged cell by cell, with all the data from the first subject preceding all the data from the second subject, and so on. Both the cells and the within-subjects scores should be arranged hierarchically (e.g., A1B1, A1B2, A1B3, A2B1, A2B2, A2B3).

Output. The program prints the cell means and the ANOVA summary table on the CRT and, optionally, on a line printer. The $\hat{\epsilon}$ statistic is reported for each within-subjects effect.

Availability. A listing of the program with detailed instructions on its use can be obtained at no cost by writing to David M. Lane, Department of Psychology, Rice University, Houston, Texas 77001. A diskette containing the program is available for $\$ 8$.

\section{REFERENCES}

Bock, R. D. Multivariate statistical methods in behavioral research. New York: McGraw-Hill, 1975.

HuYnh, H. Some approximate tests for repeated measures designs. Psychometrika, 1978, 43, 161-175.

Winer, B. J. Statistical principles in experimental design (2nd ed.). New York: McGraw-Hill, 1971.

(Accepted for publication December 19, 1980.) 